ojs.uv.es/index.php/qdef/index

\title{
Musicales traducidos y censurados en los escenarios españoles
} (1955-1985)

\author{
Raquel Merino Álvarez \\ Universidad del País Vasco, UPV/EHU \\ raquel.merino@ehu.es
}

Resumen: Esta contribución analiza, a partir del análisis del catálogo de espectáculos musicales traducidos (censurados) y representados en los escenarios españoles (TRACEmu, 1955-1985), recopilado en los archivos de censura, los casos más representativos del periodo: El hombre de la Mancha (1966), Godspell (1974), Hair y Jesucristo Superstar (1975).

Palabras clave: traducción; censura; musicales.

Abstract: The TRACEmu catalogue (Censored translations of musical productions, 1955-1985) compiled from Spanish censorship archives will serve as a starting point to analize the most representative cases of the period: Man of La Mancha (1966), Godspell (1974), Hair and Jesus Christ Superstar (1975).

Keywords: translation; censorship; stage musicals. 



\section{Teatro, traducción y censura}

En 2015 se cumplen cuarenta años de la muerte de Franco, cincuenta de la llegada de los Beatles a España y al menos sesenta desde el primer estreno de un musical importado de Broadway. Investigar, a fecha de hoy, sobre traducción (y censura) en el teatro español del siglo pasado supone abordar la historia teatral desde la perspectiva que aporta el rico legado documental dejado por la actuación burocrático-censora del régimen franquista.

Gracias a las investigaciones centradas en los archivos de censura se ha podido cartografiar el mapa de la traducción teatral en España. Pérez L. de Heredia (2005) ha investigado el periodo que va desde el final de la Guerra Civil hasta principios de la década de los sesenta, y Bandín (2007) ha analizado todo el periodo franquista centrándose en el teatro clásico inglés. La necesaria labor de catalogación de las producciones que pasaron por el filtro burocrático-censor que aportan estudios de este tipo ha permitido esbozar la historia de las traducciones (Merino 2010, 2012) para los escenarios españoles del siglo XX y acometer el estudio de géneros como el que nos ocupa: el musical.

Partiendo principalmente de datos procedentes del Archivo General de la Administración (AGA), se ha compilado el catálogo de traducciones de musicales TRACEmu, que recoge información sobre medio centenar de espectáculos musicales estrenados en los escenarios españoles desde $1955^{1}$. Del total de producciones catalogadas, la mitad corresponden a espectáculos que pasaron por las instancias censoras (1955-1978) o que se siguieron registrando hasta la reorganización del Ministerio de Cultura en 1985. La catalogación de las traducciones de musicales, conservadas en los fondos de censura, permite cartografiar el género, aportando profundidad documental y una gran amplitud de datos textuales y contextuales.

\footnotetext{
* Universidad del País Vasco, UPV/EHU. Grupo de Investigación consolidado TRALIMA, GIC12/197, Gobierno Vasco, IT728/13. Proyecto TRACE, FFI2012-39012-C0401T, MINECO.

${ }^{1}$ El grueso del catálogo TRACEmu lo ha recopilado la Dra. Pérez López de Heredia (www.ehu.es/trace).
} 


\section{Treinta años de teatro musical (censurado) en los escenarios españoles: 1955-1985}

Las investigaciones en el ámbito de la traducción (y censura) de espectáculos musicales en el siglo XX en España tienden a circunscribirse a casos concretos, obras, autores o años. Entre los estudios centrados en la censura musical del periodo franquista, cabe destacar los de Valiño $(2012)^{2}$, sobre la producción fonográfica o los de Fiuza (2009) sobre la censura de canciones. Ambos autores han utilizado los fondos del AGA para extraer datos que de otro modo no habrían podido recabar de forma sistemática. En el caso de los espectáculos musicales los archivos de censura son, también, la fuente documental principal (con frecuencia la única) que posibilita la catalogación y estudio de los musicales importados en España.

Vallejo (2004) identifica un "aparente ciclo de expansión" del musical en la escena española alrededor del "estreno" en 1997 de El hombre de la Mancha, pero advierte que "la historia del musical de Broadway en España es larga", y se remonta a la década de $1950^{3}$.

Otros autores (Mateo, 2008: 321) suelen citar el año 1975 como el del despegue del musical importado en los escenarios españoles, y son frecuentes las referencias al impacto y éxito de público que logró Jesucristo Superstar (Patterson, 2010). Antes de finalizar este año 1975, clave en la historia reciente de España, y antes incluso del fallecimiento de Franco el 20 de noviembre, nos encontramos con tres estrenos de espectáculos importados: una versión "irreverente" de la figura de Jesucristo, el controvertido primer desnudo integral (masculino y femenino)

\footnotetext{
${ }^{2}$ Valiño en su exhaustivo estudio, que toma el título de un expediente de censura musical de diciembre de 1972 ("el veneno va en dosis camufladas"), trata tanto la censura de las letras de canciones como el control moral de las portadas de los discos publicados. Valiño participa en el proyecto "Devuélveme la voz" de la Universidad de Alicante, centrado en la reconstrucción de material sonoro (radiofónico) de la época franquista y la transición (http://devuelvemelavoz.ua.es/).

${ }^{3}$ El "ciclo de expansión" en torno a 1997 se sustenta, según Vallejo, en un largo historial de importación de títulos anglosajones, aunque la convivencia entre el "musical autóctono y el de importación" no haya sido fácil. La popularidad del género musical se remonta a la revista y la zarzuela, géneros de gran tradición y éxito en España. Vallejo dice que había hasta 20 revistas en cartel en Madrid en 1951, y alguna producción como Doña Francisquita (1956) dirigida por Tamayo, que se mantuvo en escena durante diez temporadas.
} 
en una representación teatral $\left(E_{\text {quus }}^{4}\right)$, y el no menos polémico desnudo escénico del musical Hair (Álvaro, 1975: 276-278). Tal eclosión de un tipo de espectáculo largamente esperado en los escenarios españoles ciertamente marca el inicio de un nuevo periodo (Pérez Jiménez, 1993), pero también certifica la consolidación de un proceso que había comenzado unos veinte años antes. José Monleón sentenciaba, recién estrenada la década de los setenta, que "tras un periodo de larga penumbra adormecida", existía por fin "un teatro de la izquierda" (1971: 70). Apoyaba su afirmación en hitos como la creación del registro de Teatros de Cámara y Ensayo en 1955, cuyas sesiones habían supuesto la vía de entrada de temas ${ }^{5}$ y obras de autores extranjeros que revitalizaron de forma sustancial los escenarios españoles.

También en el caso de las producciones musicales es en 1955 cuando un éxito importado de Broadway (Al sur del Pacifico) entra en la escena española de la mano de José Tamayo, director con gran "olfato comercial” (García Ruiz, 2006: 99). La versión española, del dramaturgo José López Rubio, fue un rotundo éxito comercial, a pesar de las dificultades de índole económica y política propias de la época.

En la década de los sesenta, la gestión del equipo aperturista al frente de la Dirección General de Cinematografía y Teatro, dependiente del Ministerio de Información y Turismo, y la propia coyuntura socioeconómica, favorece la proliferación de espectáculos musicales (Merino, 2015). Pero a partir de 1969 se dejan notar los efectos de la gestión de un nuevo equipo ministerial, de signo involucionista, al frente de la censura de teatro. Así entre 1969 y 1974 se acumulan los expedientes de prohibición o, simplemente, no resueltos. A las peticiones de representación de musicales como Hair (1969) o Jesucristo Superstar (1972) se unen otras para producciones teatrales que no llegarán a los escenarios hasta 1974 o 1975.

A partir de 1975 nos encontramos con una sucesión de estrenos y reposiciones de espectáculos musicales que se siguieron registrando como expedientes de censura/ordenación (hasta 1978) o calificación (1979-1985), conforme la estructura ministerial cambia y se modifica

\footnotetext{
${ }^{4}$ Equus y Los chicos de la banda, junto con una obra de Antonio Gala, fueron las obras más taquilleras de 1975, superando las 600 representaciones (Álvaro, 1975).

${ }^{5}$ No en vano se da en 1955 la autorización para sesión de cámara de Té y simpatía, obra norteamericana que introduce el tema de la homosexualidad en los escenarios españoles (Merino, 2009: 137).
} 
la nomenclatura. Se genera nueva legislación a partir de la Constitución de 1978 y las reestructuraciones en el Ministerio de Cultura culminan, en lo tocante a teatro, con la definitiva creación del INAEM, Instituto Nacional de las Artes Escénicas y la Música (Real Decreto 565/1985). En mayo de 1985 cesa la actividad burocrática que aún tenía lugar siguiendo modelos antiguos, se dejan de incoar expedientes y de registrar solicitudes de calificación, y a partir de esa fecha ya no se archivan expedientes ni se depositan en los fondos de Cultura del AGA.

\subsection{El teatro musical censurado 1955-1968}

Como ya hemos indicado, el primer título localizado en los archivos de censura y registrado en el catálogo TRACEmu es Al sur del Pacífico, un musical estrenado en Broadway en 1949 y que José Tamayo, director del Teatro de la Zarzuela, pone en escena en 1955 con el prestigioso Luis Sagi-Vela al frente del elenco. La traducción corre a cargo de José López Rubio.

Luis Escobar, quien había sido director del María Guerrero, ahora al frente del Teatro Eslava, estrena The boyfriend, en el año 1961, en versión de Jaime de Armiñán. Dos años después Kiss me Kate, dirigida por Tamayo, se convierte en un "éxito muy nacional" (Llovet, 1963). Precisamente en 1963 se hacen visibles los intentos de apertura en la Sección de Cinematografía y Teatro dirigida por García-Escudero, se plasman por escrito las normas de censura y se publican en el Boletín Oficial del Estado. Se reorganizan las juntas de censura de cine y teatro, para las que se intenta contar con profesionales del medio teatral (y cinematográfico). En 1964 se estrena Golfus de Roma, dirigido por José Osuna. En 1966 llega a escena la producción del musical El hombre de la Mancha, que sirve, tras las oportunas negociaciones entre director, productor y autoridades, para dar una imagen de un teatro español abierto y homologable con los principales escenarios internacionales. Este estreno se convertirá en el segundo a nivel mundial, tras el debut en EE. UU. del musical más traducido y representado de la historia (Wasserman, 2003). En 1968 se importan otros dos espectáculos de Broadway: The Sound of Music (Sonrisas y Lágrimas), traducido por López Rubio y protagonizado por Alfredo Mayo, y Sweet Charity ( $\mathrm{Ca}$ ridad de noche) con Marujita Díaz. 


\subsection{El teatro musical censurado 1969-1974}

Superado el periodo aperturista, con la entrada de un nuevo equipo ministerial de corte más conservador (Muñoz Cáliz, 2006), comienzan a archivarse o denegarse solicitudes de representación de musicales polémicas como Hair (1969), que no llegará a estrenarse hasta 1975. Así en 1972 se abren sendos expedientes para Godspell y Jesucristo Superstar, pero las solicitudes de representación no obtendrán autorización hasta 1974 y 1975 respectivamente. Encontramos en este periodo referencias al estreno de El violinista sobre el tejado (1970, Teatro de la Zarzuela) y amplia cobertura del esperado y postergado estreno de Godspell (1974, Teatro Marquina).

Este musical basado en textos del Evangelio de San Mateo es un excelente ejemplo de las dificultades que encontraron todo tipo de espectáculos en el periodo. Los casi tres años que median entre solicitud de representación y autorización de Godspell dan fe de dichas dificultades, que se aplicaban incluso a textos como este de carácter religioso, traducido por un sacerdote (Martín Descalzo) y refrendado por la firma de un académico de la RAE afín al régimen (Pemán).

\subsection{El teatro musical censurado 1975-1982}

Comienza 1975 con el estreno, en el nuevo teatro Barceló de Madrid, de la comedia musical procedente de Broadway, Aplausos, y que interpretaban Conchita Montes, Conchita Márquez y Vicente Parra. Es solo el comienzo de un año repleto de éxitos de producciones importadas, que se suceden de forma imparable, al desmoronarse las barreras de contención del lustro anterior. El estreno escénico de la ópera rock Jesucristo Superstar tuvo lugar el 6 de noviembre de 1975, en el teatro Alcalá Palace 6 . Un "éxito arrollador" que fue ampliamente comentado por la crítica (Álvaro, 1975: 269-275). Entre los intérpretes principales estaban Camilo Sesto (involucrado en la producción), Teddy Bautista y Ángela Carrasco. El director fue Jaime Azpilicueta, quien también firmó la versión junto a Ignacio Artime.

\footnotetext{
${ }^{6}$ La película basada en el musical, y tachada de "irreverente y frívola", se había exhibido un año antes en la Semana Internacional de Cine de Valladolid (Monje, 2005: 4-5) y posteriormente en alguna sala de Madrid, donde originó “cierto escándalo provocado por un sector más papista que el Papa” (Álvaro, 1975: 269).
} 
El 14 de noviembre de 1975, "después de haber recorrido todas las grandes capitales del mundo y las principales provincias españolas"7, se presentó en el teatro Monumental de Madrid el "concierto en rock" Hair. Aunque algún miembro de la crítica apuntó que faltaban "escenas fundamentales" y que "el tan anunciado desnudo integral" quedaba anulado por "una falta de luz casi absoluta", la acogida de la crítica fue unánime y el éxito de público calificado de "total" (Álvaro, 1975: 276-278).

En 1976 se estrena en el Teatro Alcázar de Madrid Sugar, dirigida por Juan José de Arteche, y un año más tarde Yo quiero a mi mujer (en versión de Artime y Azpilicueta) con Paco Valladares. El musical de Broadway Oh, Calcutta será llevado a escena (1977, Teatro Príncipe) por Juan José Alonso Millán, director identificado con éxitos de taquilla, incluidos no pocos musicales. Procedente del West End londinense llega Elvis (1978) al Teatro Barceló y Evita (1980) de la mano del tandem Artime-Azpilicueta, con Paloma San Basilio, Mia Patterson y Patxi Andión en los papeles principales. Le siguen los musicales procedentes de Broadway Annie (1981), My Fair Lady y la reposición de Sonrisas y Lágrimas, dirigidos en 1982 por Alonso Millán. Se repone también Sweet Charity (1982, 1968'). Los estrenos de I do!, I do! (1983), West Side Story (1983), A Chorus Line (1984), Runaways (1984) y Snoopy (1985) completan esta lista de éxitos importados en su mayoría de EE. UU. A partir de 1985 son comunes las reposiciones entre las que cabe mencionar: The Rocky Horror Show (1996, 1974'), El hombre de la Mancha (1997, 1966¹) o My Fair Lady (2001, 1982¹). A directores asiduos del género como Alonso Millán o Azpilicueta, se unen otros nombres clave como el del productor Luis Ramírez, impulsor de éxitos de gran impacto en la década de los noventa y principios del siglo XXI, con quien colabora el traductor-adaptador Ignacio Artime (Morán, 2013).

\section{Directores, productores y traductores frente a censores: 1966-1975}

El caso del musical El hombre de la Mancha, dirigido por José Osuna y producido por Justo Alonso en 1966, y la gestación del estreno en 1975

\footnotetext{
${ }^{7}$ Hair se representó en 1974 bajo el titulo de Rock clásico de los 60 en Barcelona.
} 
de la ópera rock Jesuscristo Superstar, nos permiten observar cómo llegaban a escena costosas producciones musicales, tras el preceptivo proceso de autorización, en dos contextos políticos muy diferentes. De la apertura de los sesenta, se pasa a posiciones ultra-católicas en los setenta. En ambos periodos el papel de los productores y directores al frente de espectáculos importados, así como el de los traductores y adaptadores, responsables del texto, se complementan y contraponen con el de los censores y demás autoridades relacionadas con el control del teatro.

\subsection{El hombre de La Mancha. 1966}

El expediente (AGA 231/66) derivado de la tramitación de permiso para representar el musical El hombre de la Mancha es un caso singular de autorización apoyada por las autoridades encargadas de gestionar la censura en el teatro. Justo Alonso, productor, y José Osuna, director del espectáculo, negocian que sea en Madrid donde tenga lugar el segundo estreno mundial de un musical que, por su temática (Cervantes y Don Quijote son los personajes centrales), repercute en la imagen de España en el exterior. José María García-Escudero, director general de Cinematografía y Teatro, y Manuel Fraga, ministro de Información y Turismo, se propusieron con esta producción fomentar la imagen de un teatro español moderno, vivo y, sobre todo, homologable a nivel internacional. Esta actitud está en sintonía con lo que Alfonso Sastre denominó "liberalización de fachada", en la década de 1960, y que posibilitó la presencia de dramaturgos extranjeros en detrimento de los nacionales (Muñoz Cáliz, 2005: 143).

Si consultamos la documentación custodiada en el AGA sobre $E l$ Hombre de la Mancha, lo primero que llama la atención es la existencia de contactos previos a la solicitud formal de permiso de representación (18/8/1966). José Osuna, en una carta dirigida a García-Escudero, menciona "nuestra última entrevista", en la que se habló de la "obligación moral que tenemos en España de representar esta obra", en "una misión casi de cultura hispánica". En la carta subraya el riesgo empresarial que supone el montaje de un espectáculo musical y menciona la "solicitud de ayuda económica", que vendría a garantizar la viabilidad y éxito del espectáculo. A la carta se une la preceptiva instancia, suscrita por José Osuna y Justo Alonso (21/5/1966), en nombre de la producción teatral. 
El director general escribe al ministro el veintitrés de julio en relación con el "estreno de esta obra en Madrid, primera ciudad del mundo en la que se ofrece después del éxito en Nueva York". Le traslada su opinión favorable sobre la "versión muy literal" que le han remitido, indicando que las escenas en las que Cervantes aparece encarcelado por la Inquisición "serán total y absolutamente eliminadas" tal y como Osuna le ha asegurado. La documentación de tramitación de la solicitud (18 de agosto) incluye la recomendación por parte de uno de los censores de que "las efusiones de Aldonza con los criados" se vigilen en el visado de ensayo general. El 24 de agosto se expide la autorización para mayores de 18 años, sin supresiones, con la inusual clasificación de "radiable".

El expediente contiene tres textos mecanografiados en español: la primera "versión muy literal" de López Rubio, el texto adaptado que recoge las supresiones y modificaciones, y los "cantables" que se presentan en último lugar. El cotejo de dichos textos con el original parece indicar que la primera versión sigue el original inglés, sin supresiones ni adiciones; que en el segundo texto, "adaptado" siguiendo las indicaciones de las autoridades, se suprimen las referencias a la Inquisición y se pueden apreciar otras adiciones y modificaciones.

El estreno tuvo lugar el 30 de septiembre de 1966, en el Teatro de la Zarzuela de Madrid. Asistió el autor, Dale Wasserman, quien se percató de que las primeras líneas de la novela de Cervantes ("En un lugar de la Mancha...") habían sido añadidas al comienzo de la obra, y de que el tema musical principal, "El Sueño Imposible", no lo interpretaba Don Quijote-Cervantes (Luis Sagi-Vela ${ }^{9}$ ) sino Aldonza (Nati Mistral). Tras el estreno, el autor aprovechó la celebración y recepción posterior para preguntar al traductor el porqué de estos cambios que no le habían sido consultados o informados (Wasserman, 2003: 152-154). La respuesta

\footnotetext{
${ }^{8}$ El que una obra de teatro obtuviera la autorización para su retrasmisión radiofónica era excepcional pues por defecto se calificaba el grueso del teatro como "no radiable". En casos aislados, la clasificación "radiable" nos indica que se estima que el texto es totalmente inocuo y claramente autorizable. Esta clasificación es particularmente importante en el caso de la música ligera (Valiño, 2012: 53).

${ }^{9}$ Este barítono, de fama consolidada en el mundo de la Zarzuela y la ópera, ya se había retirado de los escenarios y estaba volcado en la industria fonográfica (fue presidente de prestigiosos sellos discográficos y de la Federación Nacional), pero reapareció de forma excepcional para esta producción.
} 
de López Rubio fue que el público español esperaba oír las famosas primeras líneas de El Quijote, en una producción de reminiscencias cervantinas. Respecto al cambio de asignación del tema principal la explicación que recibió fue contundente: la actriz principal, Nati Mistral, era "una estrella" (Wasserman, 2003: 153) ${ }^{10}$.

\subsection{Godspell. 1974}

A raíz de su estreno, $A B C$ publica la entrada "Godspell y otros musicales" (13/10/1974). Se dice que "a pesar de la oposición, se ha estrenado en Madrid el espectáculo musical Godspell", que "no es el primero" que se estrena "después de dificultades". Se menciona como antecedente el musical Rocky Horror Show y se citan Jesus Christ Superstar o The Boys in the Band, como ejemplos de "espectáculo-escándalo", "tan de moda en Europa y América" de inminente presentación ante el público español ${ }^{11}$.

Es muy significativo que el propio adaptador, José María Pemán, comentara el estreno del espectáculo musical a toda página en el diario $A B C$ y que incluso explicara cómo los promotores le encargaron que se ocupara de una cierta "reelección de la traducción leal y directa que ya había consumado con maestría el padre Martín Descalzo". Estima que se trataba de una "tarea fácil" dado que el $90 \%$ de la obra estaba extraída del Evangelio según San Mateo. Con todo, confiesa haber recibido alguna que otra carta de protesta de aquellos que "forman tertulia junto a la pila de agua bendita” (Pemán, 1974).

${ }^{10}$ En la versión estrenada en Madrid el 19 de octubre de 1997 en el Teatro Lope de Vega, adaptada por Nacho Artime e interpretada por Paloma San Basilio y José Sacristán; se mantienen estos cambios (Díaz Sande, 1998: 18). La manipulación del texto para la producción española de 1966 indujo al autor a establecer contratos de protección de los derechos de autor para sucesivas licencias (Wasserman, 2003). Aun así no son pocas las adaptaciones polémicas del texto que Wasserman también recoge respecto a las representaciones de Praga (2003: 155-156) o la de París (2003: 163-164), en versión de Jacques Brel.

${ }^{11}$ El $A B C(3 / 12 / 1974)$ también da cuenta de la presentación del disco, repasando "la historia española de Godspell" se dice que "desde un tiempo a esta parte los grandes espectáculos musicales parecen haber encontrado la manera de hacerse hueco en nuestras carteleras". 
El expediente abierto en 1972 (AGA 72/0360) recoge la solicitud cursada por la Compañía de Arturo González-Gil Carretero ${ }^{12}$. Los productores adjuntan una sinopsis argumental en la que comentan que la compañía responsable de la puesta en escena en París había sido recibida por el Santo Padre en el Vaticano e incluyen comentarios elogiosos de un cardenal. Dado lo "costoso del montaje" se facilitan tres ejemplares del texto y un disco con la banda sonora, que pudieran ayudar a valorar la idoneidad del espectáculo.

El informe del censor Mampaso es determinante: "se desvirtúa el relato original" con "gestos y acción casi circense", por tanto sería de aplicación la norma 14. ${ }^{a} 1$. $^{\mathrm{a}}$ que prohíbe "la representación irrespetuosa de creencias y prácticas religiosas", aunque admite que el concepto ("irrespetuoso") es subjetivo por lo que, aun expresando su opción de prohibir la obra, estima que debe someterse a las opiniones "más autorizadas" de la jerarquía eclesiástica. Un segundo censor (Vázquez Dodero) cree que podría representarse para mayores de 18 años. La junta de censura opta por trasladar el asunto a las "altas jerarquías eclesiásticas y políticas" para que determinen la "conveniencia" de ofrecer una representación con textos del Evangelio escenificados en forma de happening con fondos musicales de lo más in.

\subsection{Hair. 1975}

La primera solicitud de representación presentada a censura con vistas al estreno del musical Hair data de octubre de 1969 (AGA 69/0-0027). Este espectáculo que el traductor, Daniel Bohr, define como "comedia musical que apasiona a todos los públicos" es calificado por el censor José María Ortiz de "escandaloso", aunque esté "muy de actualidad en Londres y París", pero puntualiza que en México "determinó la expulsión de la compañía a las veinticuatro horas de su puesta en escena". El mayor reparo que subraya Ortiz es "la actitud antimilitarista (no antibélica) de los autores". Parece que el escándalo que precedía a Hair podría provocar la "desilusión" del público español si se presentara "aguado y endulzado".

\footnotetext{
${ }^{12}$ El expediente recoge varios libretos, en inglés y en español, utilizados en la gestión de las diversas solicitudes de autorización e incluso correspondencia entre la Iglesia en EE. UU. y los peticionarios.
} 
Se archivan con el expediente recortes de prensa como el que recoge la crónica de Vicente Romero (28/6/1969), enviado especial de Pueblo a Londres, quien habla de "escándalo del teatro mundial". El motivo fundamental de la polémica es el hecho de que los actores se presentan ante el público, al finalizar el primer acto, totalmente desnudos. En el expediente se dice que una obra que "escenifica la vida de una tribu de hippies en New York" con nombres como Claude, Sheila o Berger debería dejar pasar mucho tiempo para que se representara con protagonistas llamados "Pepe, Manolo y Conchita".

\subsection{Jesucristo Superstar. 1975}

La solicitud de permiso de representación del éxito musical de Lloyd Weber y Rice, Jesucristo Superstar, se registra el 9 de noviembre de 1972 (AGA 605-72). Firman la instancia en calidad de empresarios Andrés Kramer y Justo Alonso, la traducción corre a cargo de Ignacio Artime y Jaime Azpilicueta, quien también dirige la obra. El expediente se envía a la Junta Consultiva de Medios de Comunicación Social y el texto es valorado por los censores Artola y Zubiaurre, quienes emiten sendos informes. Llega al pleno de la Junta de Censura (5/2/1973), donde todos los miembros titulares emiten un informe. El recuento de votos favorables es de 9 (uno con clasificación para todos los públicos y dos para mayores de 14), frente a 3 censores que se decantan por la prohibición total, y 4 votos que establecen condiciones. Los miembros de la Junta favorables a la prohibición, o los que proponen restricciones, aducen que sería difícil "una traslación de mentalidades (de la anglosajona a la nuestra)" o que el texto sería "recusable" para "el espectador católico español”. Es una obra, opinan, "difícil de enjuiciar", "aunque en ocasiones hay "irreverencia". Los censores favorables estiman que "el texto mantiene gran fidelidad y respeto a los textos evangélicos", si bien "la escena de María Magdalena con Jesús necesita visado".

Consta el envío del expediente al Subdirector General de Teatro (5/2/1973). Hasta el 6 de febrero de 1975, fecha en la que Kramer y Alonso firman una nueva solicitud, no parece haberse archivado ningún otro documento. La junta, ahora rebautizada de "ordenación", procede a tramitar el permiso de representación (guía de censura) para mayores de 18 años, sin cortes, a favor de la empresa del Teatro Alcalá Palace, a reserva de visado de ensayo general. 


\section{Musicales traducidos en la escena española: éxitos importados de Broadway y espectáculos-escándalo}

Adolfo Marsillach afirmaba en sus memorias que "nada de lo sucedido en el teatro de este país habría sido posible" sin la aportación de directores pioneros como Luis Escobar ${ }^{13}$ y que "todos los que hicimos teatro en España somos, en parte, consecuencia de aquel trabajo" (Cornago, 2000: 77). Entre esos sucesores están José Tamay ${ }^{14}$, José Osuna y Jaime Azpilicueta, directores que promovieron el género musical en nuestro país. Del mismo modo, los adaptadores y traductores teatrales pioneros facilitaron con su labor la importación del género musical. Entre los traductores destaca el dramaturgo José López Rubio, miembro de la "otra generación del 27", hombre de teatro y cine, quien, debido a su experiencia en el Hollywood de los albores del cine sonoro (Torrijos, 2003), resultó ser un mediador ideal en la introducción de teatro musical extranjero. Entre los adaptadores cabe mencionar al dramaturgo José María Pemán, a quien, por su identificación con el régimen de Franco, se le encomendaron tareas de acomodación de textos polémicos como el de Godspell.

Espectáculos-escándalo como Godspell o Jesucristo Superstar, retenidos en un engranaje censor que se resistía a claudicar, arrasaron en los escenarios y, en buena parte debido a su halo de productos prohibidos, consiguieron llegar a todos los rincones de la geografía, hasta alcan-

\footnotetext{
${ }^{13}$ Escobar, quien había estado al frente del Teatro Nacional María Guerrero desde 1940 hasta 1952, fue el responsable de la introducción de parte de la cartelera del West End londinense en la década de los cuarenta, según el encargo del entonces director del British Council en Madrid, Walter Starkie, para contrarrestar la influencia germanófila en el teatro español. El papel de este director y traductor de obras de autores ingleses, como Priestley o Wilder, en la historia del teatro español ha quedado un tanto difuminada y marcada por su identificación con el régimen. Relacionado con el entorno de García Lorca y colaborador de La Barraca antes de la Guerra Civil, su temprano nombramiento como responsable de los teatros nacionales marcó sin embargo su carrera posterior. Escobar dirigió en los sesenta producciones musicales autóctonas como $L a$ bella de Texas (1965), "basada” en la polémica La corte del Faraón, para la que contó con Nati Mistral y con la que consiguió burlar el veto censor a la Corte.

${ }^{14}$ Tamayo dio una primera función al aire libre de La verbena de la Paloma en La Corrala de Madrid en 1954 (García Ruiz, 2006: 99) y estrenó La viuda alegre y Kiss me Kate en 1963 y Carmen de Bizet en 1964.
} 
zar un amplio espectro de público ${ }^{15}$. Sin los musicales importados que debutaron en los años cincuenta, no habríamos podido mantener una tradición posterior que ha ido creciendo y alimentándose de nuevas producciones y sonadas reposiciones hasta consolidar una cartelera repleta de productos de "genuino sabor americano" (Vallejo, 2004) que siguen "la fórmula de la empresa multinacional" (Pemán, 1974).

Como puede apreciarse, el enfoque cronológico del comienzo, así como la utilización de los fondos documentales de censura, nos permiten confirmar que hubo en España más musicales extranjeros traducidos y representados, que los que en ocasiones se ha llegado a dar por hecho. Y que los musicales importados llegaron a nuestra escena con mayor antelación de lo que se ha venido documentando.

$\mathrm{Al}$ igual que ocurrió con otros géneros teatrales, con el género musical se intentó incorporar a la escena española aquellas obras, y sobre todo tendencias, que se abrían paso en el resto del mundo occidental.

A través de los casos presentados en este artículo se puede obtener una idea clara del proceso de importación de musicales extranjeros, su problemática y los entresijos que debía seguir una obra y, con ella, todos los involucrados en el intento de hacerla llegar al público, es decir, promotores, productores, traductores y directores.

Se ha podido constatar la extraña cohabitación y el inestable equilibrio que debían mantener en ocasiones, como en el caso de El Hombre de la Mancha, la censura y el escenario, para conjugar la normativa con las urgencias económico-empresariales y la labor de ariete cultural de los profesionales, labor más o menos solapada $\mathrm{y}$, en ocasiones, hasta pactada.

\section{Bibliografía}

Álvaro, Francisco. 1975. El Espectador y la crítica. El teatro en España en 1975. Valladolid: Edición del autor.

\footnotetext{
${ }^{15}$ La "Comisión de Calificación" de obras teatrales, según el trámite de solicitud presentado por el Grupo A Posta de Valencia, calificó en 1978 Jesucristo Superstar para mayores de 14 años. Igual suerte siguieron la mayor parte de espectáculos mencionados en este articulo. Tal es el caso de Godspell, espectáculo autorizado para mayores de 14 años en 1976. De estar prohibidos (o no-autorizados) a principios de los setenta estas obras llegaron a representarse ante menores de 18 a finales de la década.
} 
Bandín, Elena. 2007. Traducción, recepción y censura de teatro clásico inglés en la España de Franco. Estudio descriptivo-comparativo del Corpus TRACEtci (1939-1985) (tesis doctoral inédita). León: Universidad de León.

Cornago, Óscar. 2000. Discurso teórico y puesta en escena en los años sesenta: la encrucijada de los realismos. Madrid: CSIC.

Díaz Sande, José Ramón. 1998. El hombre de la Mancha. Nada que envidiar a Hollywood. Reseña 290: 18. http://www.madridteatro.eu/teatr/teatro/ teatro062.htm [Acceso 05/01/2015].

Fiuza, Alexandre-Filipe. 2009. Censura y represión a los músicos españoles y portugueses en las décadas de 1960 y 1970. En Navajas, Carlos \& Iturriaga, Diego (ed.) Crisis, dictaduras, democracia. I congreso internacional de Historia de Nuestro Tiempo. Logroño: Universidad de La Rioja, 237-247.

García Ruiz, Víctor. 2006. El teatro español entre 1950 y 1955. En García Ruiz, Víctor \& Torres Nebreda, Gregorio (ed.) Historia y antología del teatro español de posguerra, vol. III. Madrid: Fundamentos, 11-170.

Llovet, Enrique. 1963 (18 de diciembre). Estreno de Kiss me Kate en el Alcázar. $A B C$, p. 103.

Mateo, Marta. 2008. Anglo-American musicals in Spanish theatres. The Translator 14(2): 319-42.

Merino Álvarez, Raquel. 2009. Building TRACE (translations censored) theatre corpus: some methodological questions on text selection. En Muñoz, Micaela (ed.) Translation and Cultural Identity: Selected Essays on Translation and Cross-Cultural Communication. Newcastle: Cambridge Scholars Publishing, 129-153.

Merino Álvarez, Raquel. 2010. La historia de las traducciones de teatro inglés en España en el siglo XX: perspectiva desde el proyecto TRACE. En Rabadán, Rosa (ed.) Lengua, traducción, recepción. En honor de Julio César Santoyo/Language, translation, reception. To honor Julio César Santoyo. León: Universidad de León, 357-384.

Merino Álvarez, Raquel. 2012. A historical approach to Spanish theatre translations from censorship archives. En García-Izquierdo, Isabel \& Esther Monzó (ed.) Iberian Studies on Translation and Interpreting. Oxford: Peter Lang, 123-140.

Merino Álvarez, Raquel. 2015. The censorship of theatre translations under Franco: the 1960s. Perspectives. Studies in Translatology. Ideology, Censorship and Translation: Past and Present. 23(4). doi: http://dx.doi. org/10.1080/0907676X.2015.1051558

Monje, Carlos. 2005 (20 de diciembre). Censores burlando la censura. Seminci 50 aniversario, $4-5$. 
Monleón, José. 1971. Treinta años de teatro de la derecha. Barcelona: Tusquets.

Morán, José. 2013 (6 de agosto). Memorias de Nacho Artime. La nueva España. http://www.lne.es/asturias/2013/08/06/infarto-productor-evitainvito-hospital/1451889.html [Acceso 05/01/2015].

Muñoz Cáliz, Berta. 2005. El teatro crítico español durante el franquismo visto por sus censores. Madrid: Fundación Universitaria Española.

Muñoz Cáliz, Berta. 2006. Expedientes de la censura teatral franquista. Madrid: Fundación Universitaria Española.

Patterson, Mia. 2010. 75 años de historia del musical en España (1930-2005). Madrid: Fundación Autor.

Pemán, José María. 1974 (24 de octubre). Godspell. ABC, p. 3.

Pérez Jiménez, Manuel. 1993. La escena madrileña en la Transición Política (1975-1982). Universidad de Alcalá de Henares. http://hdl.handle. net/10017/763 [Acceso 05/01/2015].

Pérez López de Heredia, María. 2005. Inventario de las traducciones censuradas de teatro norteamericano en la España de Franco (1939-1963). En Merino, Raquel; Santamaría, José Miguel \& Pajares, Eterio (ed.) Trasvases culturales: literatura, cine y traducción, 4. Bilbao: Universidad del País Vasco, 97-112. http://hdl.handle.net/10810/10537 [Acceso 05/01/2015].

Torrijos, José María (ed.) 2003. José López Rubio. La otra generación del 27. Discurso y cartas. Madrid: Centro de Documentación Teatral.

Valiño, Xavier. 2012. Veneno en dosis camufladas. La censura en los discos de pop-rock durante el franquismo. Milenio: Barcelona.

Vallejo, Javier. 2004 (9 de octubre). El original y la copia. Babelia. El País. http://elpais.com/diario/2004/10/09/babelia/1097276779_850215.html [Acceso 05/01/2015].

Wasserman, Dale. 2003. The impossible musical. The "Man of La Mancha" Story. Nueva York: Applause. Theatre and Cinema books. 
